\title{
Bio-medical entity prioritisation based on literature with Semantic Web annotations
}

\author{
Norio Kobayashi \\ From Biomedical Linked Annotation Hackathon 2015 \\ Kashiwa, Japan. 23-27 February 2015
}

\begin{abstract}
Summary
An extension of the General and Rapid Association Study Engine (GRASE), a Semantic Web data (entity) prioritisation engine, is discussed. The GRASE employs a unique mechanism to prioritise entities using entitydocument relations by computing the statistical significance between entities and user keywords based on the number of related documents. We describe an improvement of prioritisation accuracy and connectivity to the Semantic Web using PubAnnotation.
\end{abstract}

\section{Introduction}

In life-science data analysis, prioritisation of entities among a large number of candidates is an important task. The General and Rapid Association Study Engine (GRASE) [1] introduced here is a Semantic Web data prioritisation engine. The GRASE was originally used for causative gene search in a RIKEN mouse ENUmutagenesis program and 65 genes were successfully highly ranked [2] through its web interface called PosMed [3]. The supported data has been extended and used successfully to discover bioresources in mice and Arabidopsis. The unique characteristic of GRASE prioritisation is employing entity-document relations where documents include MEDLINE abstracts. This mechanism has an advantage in which each prioritised entity can be shown with related documents as evidence, and these entities can be searched even if their descriptions are not given as Semantic Web data. However, the precision of such functions depends strongly on the accuracy of the entity-document relations. In the following, we examine the GRASE with a PosMed example and propose an extension using PubAnnotation [4] to

Correspondence: norio.kobayashi@riken.jp

Advanced Center for Computing and Communication (ACCC), RIKEN, Wako, Japan 


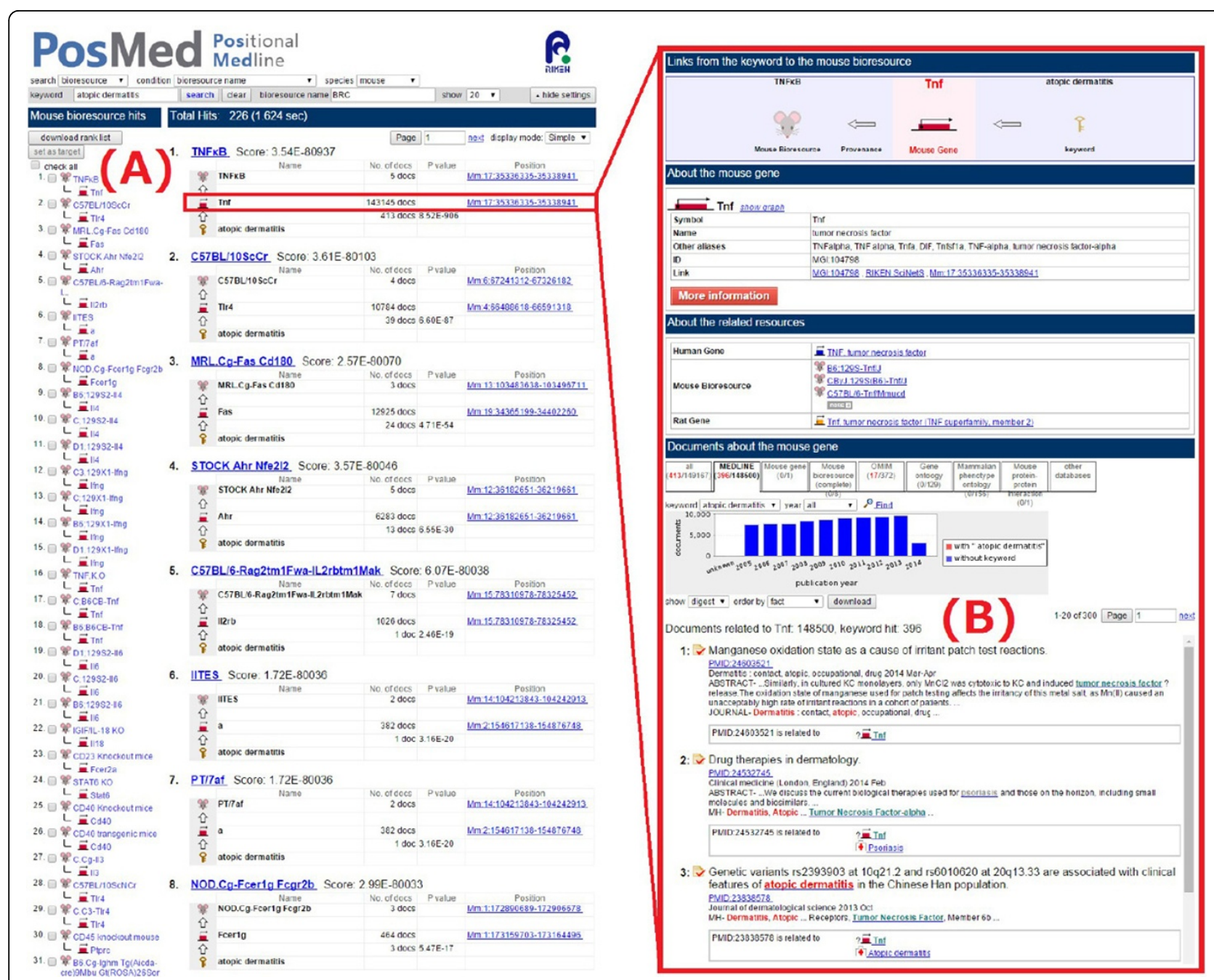

Figure 1 PosMed search example of ranked mouse bioresources with keyword 'atopic dermatitis'. (A) Resultant ranking of bioresources; (B) list of documents (including MEDLINE abstracts and OMIM) associated with the mouse genome informatics (MGI)-mouse gene [6] Tnf connected by a semantic link to bioresource TNF $\kappa$ B (ranked first). Documents associated with the user keyword, terms associated with TNF $\kappa$ B and terms associated with other entities are highlighted in red, green and grey, respectively.

\section{Association between literature and entities}

In the current implementation, entity-document relations are obtained by full-text search with humancurated logical queries defined as a list of entity names concatenated with logical operators such as AND, OR and NOT. For example, the logical query for the Arabidopsis gene AT1G03880 (cruciferin B, CRB) is defined as follows:

\section{(AT1G03880 OR CRU2 OR CRB OR 'CRUCIFERIN 2' OR 'CRUCIFERIN B') \\ AND (Arabidopsis) NOT (' chloroplast RNA binding') .}

Logical queries for mouse, human and Arabidopsis genes have already been curated manually.

\section{Discussion}

Here, we discuss GRASE statistical prioritisation search, which ranks entities using entity-document relations. Although our curation method to obtain entity-document relations is effective for frequently updated arbitrary document sets, it may still produce false-positive errors and cannot be extended for additional entities. The open collections of annotations of literature, including PubAnnotation can be used to solve this problem, which allows GRASE search to be performed more precisely. Another advantage of introducing PubAnnotation is the ability to show documents with detailed annotation with semantic links.

Therefore, future work includes implementing an interface to input/output PubAnnotation data. More concretely, we would like to introduce PubAnnotation 
data as accurate entity-document relations in addition to our query approach and implement functions to display and download documents that are related to the resultant entity in PubAnnotation format.

\section{Acknowledgements}

Part of this research was supported by RIKEN Incentive Research Projects. We thank Dr. Hiroshi Masuya for his useful comments regarding bioresource ranking.

Published: 6 August 2015

\section{References}

1. Kobayashi N, Toyoda T: Statistical search on the Semantic Web. Bioinfom 2008, 24(7):1002-1010.

2. Masuya H, Yoshikawa S, Heida N, Toyoda T, Wakana S, Shiroishi T: Phenosite: a web database integrating the mouse phenotyping platform and the experimental procedures in mice. J Bioinform Comput Biol 2007, 5:1173-1191.

3. Makita Y, Kobayashi N, Yoshida Y, Doi K, Mochizuki Y, Nishikata K, Matsushima A, Takahashi S, Ishii M, Takatsuki T, Bhatia R, Khadbaatar Z, Watabe H, Masuya H, Toyoda T: PosMed: Ranking genes and bioresources based on Semantic Web Association Study. Nucleic Acids Res 2013, 41(Web Server):W109-W114.

4. PubAnnotation: Share your annotation in alignment with others. [http:// pubannotation.org/].

5. Eppig JT, Strivens M: Finding a mouse: the International Mouse Strain Resource (IMSR). Trends Genet 1999, 15(2):81-82.

6. Blake JA, Bult CJ, Eppig JT, Kadin JA, Richardson JE, Mouse Genome Database Group: The Mouse Genome Database: integration of and access to knowledge about the laboratory mouse. Nucleic Acids Res 2014, 42(Database):D810-D817

doi:10.1186/1753-6561-9-S5-A6

Cite this article as: Kobayashi: Bio-medical entity prioritisation based on literature with Semantic Web annotations. BMC Proceedings 2015 9(Suppl 5):A6.

\section{Submit your next manuscript to BioMed Central} and take full advantage of:

- Convenient online submission

- Thorough peer review

- No space constraints or color figure charges

- Immediate publication on acceptance

- Inclusion in PubMed, CAS, Scopus and Google Scholar

- Research which is freely available for redistribution

Submit your manuscript at www.biomedcentral.com/submit
Biomed Central 